\title{
EVALUACIÓN DE MATERIALES FILTRANTES PARA EL REÚSO EN AGRICULTURA DE AGUAS RESIDUALES TRATADAS PROVENIENTES DE ZONAS ÁRIDAS
}

\author{
ASSESSMENT OF FILTERING MATERIALS FOR WATER REUSE IN \\ AGRICULTURE OF TREATED WASTEWATER FROM ARID AREAS
}

Ismael Leonardo Vera Puerto', Mauricio Rojas Arredondo², Wladimir Chávez Yavara ${ }^{3}$, Bernardo Tomás Arriaza Torres ${ }^{4}$

Fecha de recepción: 18 de Junio de 2015

Fecha de aprobación: 10 de Diciembre de 2015

Referencia: I. L. Vera Puerto, M. Rojas Arredondo, W. Chávez Yavara, B. T. Arriaza Torres (2016). Evaluación de materiales filtrantes para el reúso en agricultura de aguas residuales tratadas provenientes de zonas áridas. Ciencia e Ingeniería Neogranadina, 26 (1), pp. 5-19, DOI: http://dx.doi.org/10.18359/rcin.1633

\section{RESUMEN}

Este trabajo tuvo por objetivo evaluar la arena (A), el carbón activado (CA) y la zeolita (Z) como materiales filtrantes para valorar su efecto en reutilización para agricultura de aguas residuales tratadas de un ambiente árido. Para esto se construyeron 7 columnas de filtración: a) columna 1, $100 \%$ de $Z$, b) columna $2,100 \%$ de CA (origen A), c) columna 3, $100 \%$ de CA (origen B), d) columna $4,100 \%$ de $A$, e) columna $5,50 \%$ de $Z$ sobre $50 \%$ de CA, f) columna $6,50 \%$ de $A$ sobre $50 \%$ de CA, y g) columna 7, $50 \%$ de $Z$ sobre $50 \%$ de $A$. En el afluente y efluente se evaluó boro (B), cloruro $\left(\mathrm{Cl}^{-}\right)$, sulfato $\left(\mathrm{SO}_{4}^{-2}\right)$, calcio $\left(\mathrm{Ca}^{+2}\right)$, conductividad eléctrica (CE), pH y temperatura (T), junto a un grupo de metales pesados (As, Cd, Cu, Fe, Mn, Pb y Zn). Los resultados mostraron cómo el CA (de origen A o B) es capaz de remover boro a valores por debajo de 0,75 mg/L. Sin embargo, en una etapa inicial (primera quincena) los efluentes a este medio filtrante, presentaron un valor de CE mayor

1. Ing. Civil, Magíster en Hidrosistemas, Doctor en Ciencias Ambientales. Investigador en Tecnologías Ambientales, Centro de Investigación y Desarrollo en Recursos Hídricos (CIDERH), Universidad Arturo Prat, Iquique, Chile, leonardo.vera@ciderh.cl

2. Técnico Analista Químico. Técnico de Laboratorio, Centro de Investigación y Desarrollo en Recursos Hídricos (CIDERH), Universidad Arturo Prat, Iquique, Chile, laboratorio@ciderh.cl

3. Ing. Civil Ambiental. Profesional de Investigación, Centro de Investigación y Desarrollo en Recursos Hídricos (CIDERH), Universidad Arturo Prat, Iquique, Chile,wladimir.chavez@ciderh.cl

4. Antropólogo Físico, Doctor en Antropología. Director Instituto de Alta Investigación, Universidad de Tarapacá, Arica, Chile, barriaza@uta.cl 
a 2,5 dS/m, y de $\mathrm{pH}$ mayor a 8,4, lo que seguramente traerá problemas para su aplicación en riego de cultivos. Por su parte, la arena, al ser de la región, presenta problemas de lixiviación de elementos, como el arsénico y el sodio, por lo que no debería utilizarse como material filtrante. Por el contrario, el único medio evaluado que tiene potencial de ser aplicado en la reducción de la salinidad es la zeolita. Este material filtrante, sin mezcla con otro, fue capaz de reducir hasta un $20 \%$ el valor de la CE sin tener un efecto negativo en ninguno de los otros parámetros evaluados en este estudio, y además, sin mostrar un estado de saturación durante el tiempo de operación evaluado. En el caso de las columnas que utilizaron mezclas de materiales, no existe un efecto sinérgico para la eliminación de los parámetros de calidad del agua evaluados.

Palabras clave: agua residual municipal, arena, carbón activado, filtración, salinidad, zeolita.

\section{ABSTRACT}

This study was aimed to evaluate Sand (A), Activated Charcoal (CA) and Zeolite (Z) as filter materials to assess their effect on agricultural water reuse of municipal treated wastewater coming from arid environments. For this, it was built 7 filtration columns: a) column 1, $100 \%$ of $Z$, b) column 2, $100 \%$ of CA coming from $A$, c) column 3, $100 \%$ of CA coming from $B$, d) column $4,100 \%$ of $A$, e) column 5 , $50 \%$ of $Z$ above $50 \%$ of CA, f) column $6,50 \%$ of A above $50 \%$ of CA, and g) column $7,50 \%$ of $Z$ above $50 \%$ of $A$. In influent and effluents were evaluated the following parameters, Boron (B), Chloride $\left(\mathrm{Cl}^{-}\right)$, Sulfate $\left(\mathrm{SO}_{4}{ }^{-2}\right)$, Calcium (Ca+2), Electrical Conductivity (EC), $\mathrm{pH}$, Temperature (T), besides a group of heavy metals (As, Cd, Cu, Fe, Mn, Pb y Zn). The results showed that Activated Charcoal (coming from A or B) removes Boron to concentrations below $0.75 \mathrm{mg} / \mathrm{L}$. However, during initial stage (first two weeks) effluents from Activated Charcoal filters showed EC above $2.5 \mathrm{dS} / \mathrm{m}$ and $\mathrm{pH}$ above 8.4. Probably these effluents will bring problems in water reuse for irrigation in crops. In the case of Sand, lixiviation problems with Arsenic and Sodium were defined. This lixiviation problem was associated to material's origin. By contrast, the Zeolite is the only material with potential use as filter to remove salinity. The Zeolite (no mixing with other filter material) was able to reduce by up to $20 \%$ the value of the EC without negative effect in any other water quality parameter evaluated in this study. In addition, Zeolite did not show any saturation problem during operational time. In the case of columns with different materials, synergy effect is not present for removal of the different water quality parameters evaluated in this study.

Keywords: municipal wastewater, sand, activated charcoal, filtration, salinity, zeolite.

\section{INTRODUCCIÓN}

La escasez de agua dulce se ha convertido en un problema creciente a nivel mundial [1]. En zonas áridas con una disponibilidad hídrica inferior a $1050 \mathrm{~m}^{3}$ /(hab.-año) (50\% del promedio mundial) [2], el conflicto por acceso a fuentes de agua dulce es creciente. Es por esto que se hace necesario tener fuentes alternativas. Entre las diferentes alternativas, 
la reutilización de aguas residuales municipales tratadas representa una fuente utilizada en ambientes áridos, por ejemplo Israel, donde más del $60 \%$ de las aguas residuales municipales son reutilizadas [3].

Actualmente, la agricultura ocupa más del 70\% de los recursos de agua dulce para riego, y en zonas como California en los Estados Unidos, cerca del $50 \%$ de las aguas reutilizadas son destinadas para este fin [1-3-4]. Por tanto, las aguas residuales urbanas tratadas aplicadas al riego representan un campo importante para trabajar en países en vías de desarrollo en general. En el caso de Chile, las coberturas de tratamiento a nivel secundario de zonas urbanas superan el $90 \%$. Sin embargo, menos del $5 \%$ de las Plantas de Tratamiento de Aguas Residuales Municipales reutilizan sus efluentes en riego de actividades agrícolas, situación que sería similar a la de otros países en vías de desarrollo [5].

Al respecto, la calidad de las aguas aplicadas a riego ha sido definida en diversos manuales internacionales, y en el caso de Chile, el tema está regulado por la Norma Chilena 1333 of. 1987 [3-6-7-8]. Los parámetros regulados están basados en cuatro grandes grupos: a) físicos, b) orgánicos, c) salinidad y d) metales pesados [9]. Considerando que en general los procesos comúnmente aplicados al tratamiento de aguas residuales municipales son capaces de adecuar parámetros físicos, y eliminar materia orgánica y sólidos bajo los niveles de las diversas legislaciones, el problema de calidad del agua a reutilizar en riego en zonas áridas se centra principalmente en la salinidad y los metales pesados [10-11].

La salinidad ha sido definida como uno de los parámetros más importantes que afecta la producción de los cultivos, y que por tanto debe ser controlada y monitoreada cuando se emplea agua residual municipal tratada para riego [3]. La salinidad es indicativa de la cantidad de minerales disueltos en el agua. Se valora de forma continua por el macro parámetro Conductividad Eléctrica (CE), y de forma complementaria por la Tasa de Adsorción de Sodio (TAS). Su importancia radica en que valores de CE por sobre los $3 \mathrm{dS} / \mathrm{m}$ [6], influyen en la presión osmótica del agua en el suelo, y por lo tanto, conducen a un mayor gasto de energía por parte de la planta para extraer el agua. Esto trae como resultado, que la respiración de las plantas aumente, afectando el crecimiento y rendimiento de la mayoría de cultivos [9]. En este contexto diversas tecnologías, como la ósmosis inversa, el uso de membranas, entre otras, han sido aplicadas para la eliminación del contenido de sales del agua [12]. Sin embargo, la mayor desventaja de estos métodos de eliminación de sales ha sido el alto costo de implementación y el alto consumo de energía [13]. Por esta razón, a fin de reducir el contenido de sales en el agua, tecnologías alternativas como la filtración han sido aplicadas [12-13].

Varios materiales pueden ser usados como medios de filtración para adsorción de compuestos durante el tratamiento de aguas residuales, entre los que se destacan la zeolita natural y el carbón activado [14-15]. La zeolitas naturales son aluminio silicatos con una estructura basada en $\mathrm{AlO}_{4}$ y $\mathrm{SiO}_{4^{\prime}}$ que poseen una capacidad de intercambio catiónico alta, así como propiedades de tamiz molecular, características útiles para la eliminación de sustancias del agua residual [14]. Por otro lado, el carbón activado es un producto comercial con superficie específica alta, de carácter microporoso y una alta capacidad de adsorción, por lo que elimina una amplia variedad de contaminantes orgánicos e 
inorgánicos y metales pesados presentes en medios acuosos [12].

Por tanto, y dada la amplia facilidad de consecución de zeolita natural y carbón activado, este trabajo ha tenido por objetivo evaluar mediante ensayos en columnas, la aplicabilidad de utilizar estos materiales filtrantes en el control de la salinidad del agua residual urbana efluente a un sistema de tratamiento emplazado en una zona árida, y además, evaluar la influencia de esta filtración en otros parámetros de importancia en la calidad del agua a reutilizarse en riego de actividades agrícolas.

\section{MATERIALES Y MÉTODOS}

\subsection{Afluente}

Se trabajó con agua residual municipal efluente a una Planta de Tratamiento que sirve a una comunidad de cerca de 100.000 habitantes y está localizada en un ambiente árido. El proceso de tratamiento consiste de rejillas para eliminación de sólidos, lagunas aireadas mecánicamente por aireadores superficiales, y un sistema de desinfección con cloro. El afluente para los experimentos fue tomado en la salida general de la Planta de Tratamiento de Aguas Servidas (PTAS). Este fue almacenado en contenderos plásticos de $20 \mathrm{~L}$ y mantenido en cámara oscura a una temperatura de $4^{\circ} \mathrm{C}$ por máximo tres semanas.

\subsection{Sistema experimental}

Se construyeron 7 columnas de filtración con tubería PVC sanitaria gris de $75 \mathrm{~mm}$. Estas tuvieron una longitud total de 0,8 m. Su configuración de arriba hacia abajo fue la siguiente: a) 0,1 m de borde libre, b)
0,1 m con grava de $19 \mathrm{~mm}, \mathrm{c})$ 0,50 m con el material filtrante, y d) 0,1 m con grava de 19 $\mathrm{mm}$. Como material filtrante se usó: a) zeolita natural (Z),b) carbón activado, de dos orígenes diferentes (CA-A y CA-B), y c) arena (A) de la quebrada Juan Morales y Sagasca, localizada en la región de Tarapacá al norte de Chile. La Tabla 1 resume la composición química de los materiales utilizados en el experimento.

De las 7 columnas, 4 fueron construidas con un solo material filtrante, mientras que las 3 restantes se construyeron con una combinación de los materiales filtrantes, de la siguiente forma: a) columna con zeolita (Z), b) columna con carbón activado (CA-A), c) columna con carbón activado (CA-B), d) columna con arena $(A)$, e) columna con $50 \%$ de zeolita en la parte superior y $50 \%$ de carbón activado en la parte inferior (Z+CA-B), f) columna con $50 \%$ de arena en la parte superior y $50 \%$ de carbón activado en la parte inferior $(A+C A-B)$, y g) columna con $50 \%$ de zeolita en la parte superior y $50 \%$ de arena en la parte inferior $(Z+A)$. El objetivo de combinar materiales en las columnas fue evaluar si existían efectos sinérgicos entre estos. La Figura 1 presenta un resumen de las columnas utilizadas para experimentación. Las columnas fueron instaladas en el laboratorio del Centro de Investigación y Desarrollo en Recursos Hídricos (Ciderh) en el campus Huayquique de la Universidad Arturo Prat, a temperatura ambiental, en la ciudad de lquique, localizada en la parte norte del desierto de Atacama. Este tipo de columnas para evaluación de capacidad de adsorción se proponen de forma similar a las usadas por [16-17].

\subsection{Estrategia de operación y monitoreo}

Cada columna se operó a una carga hidráulica de $100 \mathrm{~L} /\left(\mathrm{m}^{2}-\mathrm{d}\right)$ por 60 días. La selección de 
Tabla 1. Composición química (por microscopía electrónica de barrido acoplada a un detector de rayos X [EDX]) para cada uno de los materiales empleados en el sistema experimental. Z: zeolita; CA-A: carbón activado A; CA-B: carbón activado $B ; A$ : arena

\begin{tabular}{|c|c|c|c|c|c|}
\hline \multirow{3}{*}{ Elemento } & \multirow{3}{*}{ Compuesto } & \multicolumn{4}{|c|}{ Material } \\
\hline & & Z & CA-A & CA-B & A \\
\hline & & Composición (\%) & Composición (\%) & Composición (\%) & Composición (\%) \\
\hline Silicio & $\mathrm{SiO}_{2}$ & 64,19 & - & - & 66,10 \\
\hline Titanio & $\mathrm{TiO}_{2}$ & 0,52 & - & - & 0,68 \\
\hline Aluminio & $\mathrm{Al}_{2} \mathrm{O}_{3}$ & 11,65 & - & - & 14,68 \\
\hline Hierro & $\mathrm{Fe}_{2} \mathrm{O}_{3}$ & 2,53 & - & - & 5,02 \\
\hline Manganeso & $\mathrm{MnO}$ & 0,03 & - & - & - \\
\hline Magnesio & MgO & 0,66 & - & - & 2,30 \\
\hline Calcio & $\mathrm{CaO}$ & 3,42 & - & - & 2,49 \\
\hline Sodio & $\mathrm{Na}_{2} \mathrm{O}$ & 0,75 & 0,04 & 0,04 & 2,71 \\
\hline Potasio & $\mathrm{K}_{2} \mathrm{O}$ & 1,60 & 0,17 & 0,17 & 4,12 \\
\hline Fósforo & $\mathrm{P}_{2} \mathrm{O}_{5}$ & 0,03 & - & & - \\
\hline Carbono & PXC & 14,64 & - & & - \\
\hline Carbono & $\mathrm{CO}_{2}$ & - & 99,72 & 99,78 & - \\
\hline Azufre & $\mathrm{SO}_{3}$ & - & - & - & 1,90 \\
\hline
\end{tabular}

Fuente: Elaboración propia.

la carga está basada en las recomendaciones de diseño para filtros de arena propuesta en Usepa [18]. Todas las columnas fueron alimentadas una vez al día durante todo el tiempo de operación.

Para evaluar parámetros de calidad de agua relacionados con cationes y aniones, que tienen influencia en la CE y que son importantes en la reutilización de aguas residuales, los días 0,20 , 40 y 60 , se midieron en los efluentes a las 7 columnas, los parámetros: a) boro (B), b) calcio $\left(\mathrm{Ca}^{+2}\right)$, c) cloruro $\left(\mathrm{Cl}^{-}\right)$y d) sulfato $\left(\mathrm{SO}_{4}^{-3}\right)$. Para evaluar el efecto de la reducción de la salinidad se midió tres veces por semana la conductividad eléctrica (CE), y junto a esta, parámetros físicos como el pH y la temperatura (T). Finalmente, al día 0 y al día 60 de operación, se evaluaron otros parámetros de interés (principalmente metales) para reutilización en riego agrícola, como el As, Cd, Cu, Fe, Mn, Pb, Zn y la Tasa de Adsorción de Sodio (TAS).

\subsection{Métodos analíticos}

Para los análisis químicos de calidad del agua, las muestras fueron filtradas con filtros de fibra de vidrio de tamaño de poro 


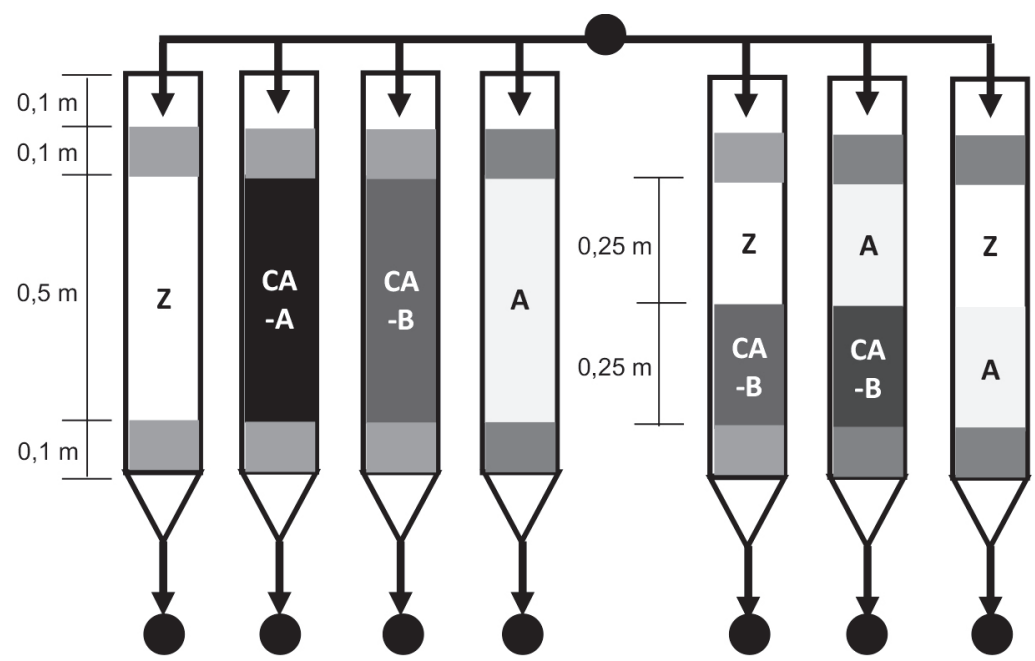

Figura 1. Columnas de experimentación.

Punto de toma de muestra. A: arena; CA-A: carbón activado - A; CA-B: carbón activado - B; Z: zeolita

Fuente: Elaboración propia.

de $0,45 \mu \mathrm{m}$. El B, $\mathrm{Ca}^{+2}, \mathrm{Cl}^{-}$y $\mathrm{SO}_{4}^{-3}$, fueron determinados de forma fotométrica a través del espectrofotómetro Merck Pharo 300 empleando test en cubetas de la marca Merck con códigos: a) $\mathrm{B}, 1.00826 .0001$, b) $\mathrm{Ca}^{+2}$, 1.00858 .0001 , c) $\mathrm{Cl}^{-}, 1.14730 .0001$, d) $\mathrm{SO}_{4}^{-3}$, 1.14548.0001. Estos métodos simplificados están basados en métodos estandarizados en APHA-AWWA-WEF [19]. La CE, el pH y la T se evaluaron con electrodo, empleando el equipo multiparamétrico portátil de marca Hanna con código de producto HI-9829.

En el caso de los parámetros As, Cd, Cu, Fe, $\mathrm{Mn}$, $\mathrm{Pb}, \mathrm{Zn}$ y TAS, las muestras fueron tomadas y enviadas al laboratorio de calidad de aguas de la empresa sanitaria local. El As, Cd, Cu, Fe, Mn, $\mathrm{Pb}$ y $\mathrm{Zn}$ fueron evaluados por procedimientos establecidos en las Normas Chilenas NCh 2313 Of. 9 y 10 [20] (INN, 1996). La TAS fue evaluada por procedimientos descritos en APHA-AWWA-WEF [19].
La composición química de los materiales usados para filtración fue llevada a cabo mediante microscopía electrónica de barrido (SEM EVO LS-10) acoplado a un detector de EDX (software INCA), en el laboratorio de Bioarqueología del Instituto de Alta Investigación en la Universidad de Tarapacá.

\section{RESULTADOS Y DISCUSIÓN}

\subsection{Características del afluente}

La Tabla 2 presenta los valores de concentración media para los diferentes parámetros caracterizados durante el tiempo de experimentación. Los valores medios de $\mathrm{pH}, \mathrm{As}, \mathrm{Cd}, \mathrm{Cu}, \mathrm{Fe}, \mathrm{Mn}$, y $\mathrm{Pb}$ pueden ser considerados como normales para los efluentes de Plantas de Tratamiento de Aguas Residuales Municipales [7]. Por el contrario, los valores medios de $\mathrm{CE}, \mathrm{B}, \mathrm{Ca}^{+2}, \mathrm{Cl}^{-}, \mathrm{SO}_{4}^{-2}$, y TAS, 
presentan valores que superan hasta en dos veces los valores de los efluentes de plantas que tratan aguas residuales urbanas típicas, pero están acordes con valores reportados para efluentes de plantas de tratamiento localizadas en ambientes áridos [7-10-11]. Estos valores se explican principalmente por la configuración geomorfológica existente en el norte de Chile, dado que los acuíferos utilizados para el abastecimiento de agua potable poseen una fuerte presencia de minerales, lo que conlleva, por ejemplo, a valores de CE cercanos a $1,5 \mathrm{dS} / \mathrm{m}$, y por tanto, a valores elevados en las aguas residuales [10].

Tabla 2. Concentración media por parámetro en el afluente

\begin{tabular}{|c|c|c|}
\hline Parámetro & Unidad & Promedio \\
\hline $\mathrm{pH}$ & Unid. & 7,74 \\
\hline $\mathrm{T}$ & ${ }^{\circ} \mathrm{C}$ & 22,5 \\
\hline $\mathrm{CE}$ & $\mathrm{dS} / \mathrm{m}$ & 2,32 \\
\hline $\mathrm{B}$ & $\mathrm{mg} / \mathrm{L}$ & 2,10 \\
\hline $\mathrm{Ca}^{+2}$ & $\mathrm{mg} / \mathrm{L}$ & 83,05 \\
\hline $\mathrm{Cl}^{-}$ & $\mathrm{mg} / \mathrm{L}$ & 277,3 \\
\hline $\mathrm{SO}{ }_{4}^{-2}$ & $\mathrm{mg} / \mathrm{L}$ & 379,8 \\
\hline $\mathrm{As}$ & $\mathrm{mg} / \mathrm{L}$ & 0,021 \\
\hline $\mathrm{Cd}$ & $\mathrm{mg} / \mathrm{L}$ & $<0,01$ \\
\hline $\mathrm{Cu}$ & $\mathrm{mg} / \mathrm{L}$ & 0,05 \\
\hline $\mathrm{Fe}$ & $\mathrm{mg} / \mathrm{L}$ & 0,49 \\
\hline $\mathrm{Mn}$ & $\mathrm{mg} / \mathrm{L}$ & 0,01 \\
\hline $\mathrm{Pb}$ & $\mathrm{mg} / \mathrm{L}$ & $<0,05$ \\
\hline Zn & $\mathrm{mg} / \mathrm{L}$ & 0,21 \\
\hline $\begin{array}{c}\text { Tasa de Adsorción } \\
\text { de Sodio (TAS) }\end{array}$ & - & 9,4 \\
\hline
\end{tabular}

Fuente: Elaboración propia. $\mathrm{n}=4$ para $\mathrm{pH}, \mathrm{T}, \mathrm{CE}, \mathrm{B}, \mathrm{Ca}_{1} \mathrm{Cl}^{-}$, $\mathrm{SO}_{4}{ }^{-2} ; \mathrm{n}=2$ para As, Cd, Cu, CaCO3, Fe, Mn, Pb, TAS, Zn.

\subsection{Comportamiento del $\mathrm{B}, \mathrm{Cl}^{-}, \mathrm{SO}_{4}^{-2}, \mathrm{Ca}^{+2}$}

La Figura 2 presenta, en diagramas de barra, las concentraciones medias típicas para boro (B), cloruro $\left(\mathrm{Cl}^{-}\right)$y sulfato $\left(\mathrm{SO}_{4}^{-2}\right)$, encontradas en los efluentes a los 7 sistemas de filtración evaluados.

La Figura 2 a) y b) muestra cómo para el caso del $B$, se presentan valores medios de concentración por debajo de 0,3 mg/L para los efluentes de las columnas que utilizaron únicamente carbón activado como medio filtrante, y por debajo de 0,5 mg/L para aquellas columnas en las que el carbón activado se mezcló con otros materiales filtrantes. Este resultado muestra que no existe interferencia ni efecto sinérgico por la mezcla del carbón activado con otros materiales. Además, la eliminación de B con sustratos orgánicos (como el carbón activado) se explica, ya que el B es absorbido por el sustrato orgánico gracias a un mecanismo de intercambio de ligando, que ocurre ya sea con un único ion hidroxilo o con más de uno de estos [21]. Tomando en cuenta lo anterior, los resultados obtenidos muestran, también, que a una tasa de aplicación de $100 \mathrm{~L} /\left(\mathrm{m}^{2}-\mathrm{d}\right)$ y una concentración influente de 2 mg/L (Tabla 1), el sistema no presenta problemas de saturación del medio en 60 días de operación. Esto es importante porque muestra cómo el carbón activado puede ser utilizado por al menos 60 días en la eliminación de $\mathrm{B}$ a niveles bajo los $0,75 \mathrm{mg} / \mathrm{L}$, que es el límite establecido por la NCh 1333 Of. 78 [8]. De acuerdo con Ayers y Westcot [6], la mayoría de cultivos presentará problemas si se riegan con aguas residuales tratadas que presentan concentraciones de B por sobre los $2 \mathrm{mg} / \mathrm{L}$ (valor efluente del sistema de tratamiento actual). Por tanto, para proyectos de reutilización basados en esta agua residual tratada podrían definirse dos 

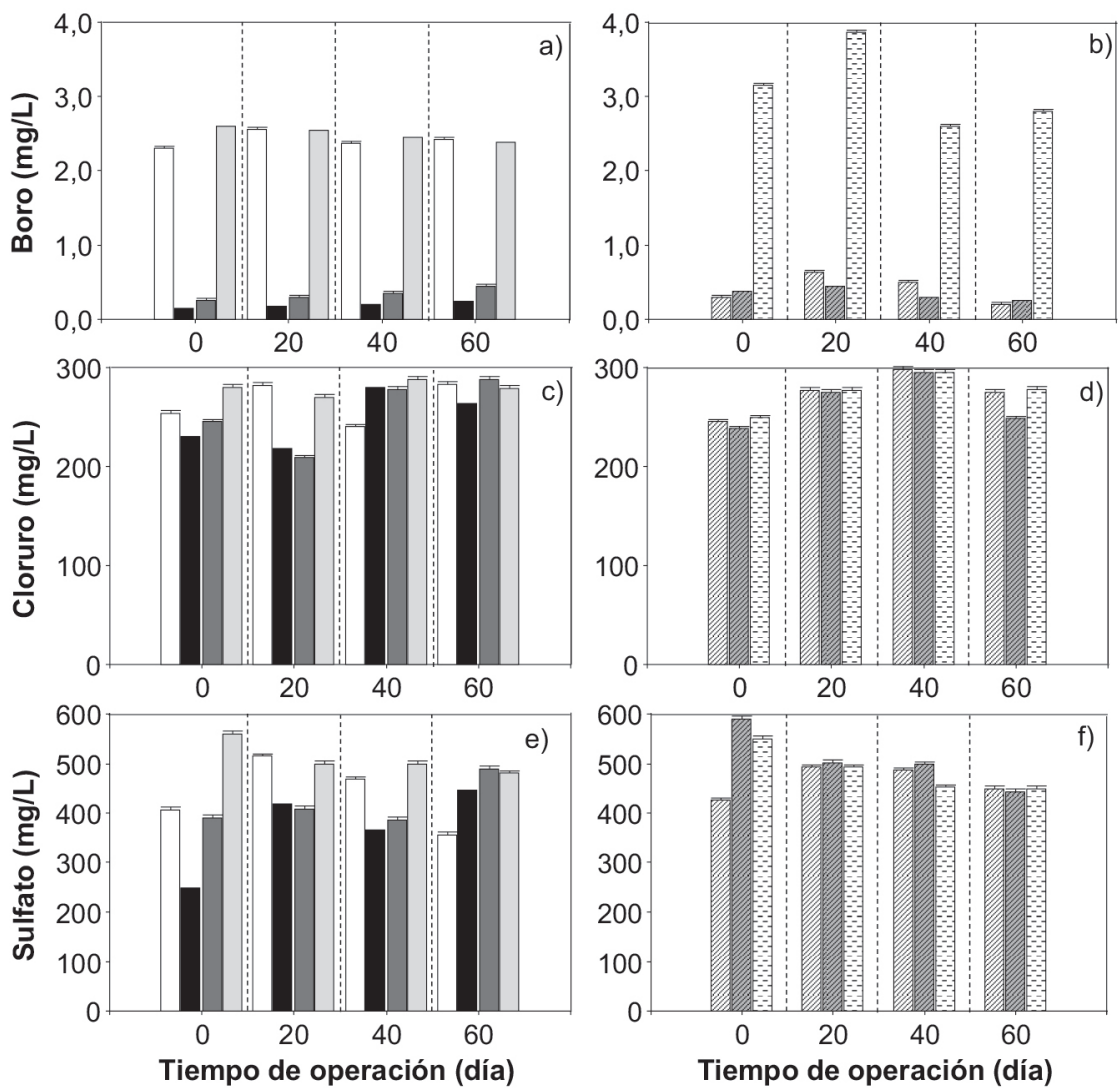

Figura 2. Comportamiento de B efluente a los sistemas de filtración. a), c) y e). Filtros de un solo material: $(\square) Z_{1}(\mathbf{\square}) C A$ -

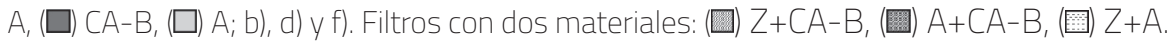

Fuente: Elaboración propia.

alternativas: a) reducir el $\mathrm{B}$ bajo los $0,75 \mathrm{mg} / \mathrm{L}$, o b) seleccionar cultivos como lechuga, repollo, apio, avena, que tienen una tolerancia mayor al $B$, pero con el cuidado de evaluar la influencia de otros parámetros de calidad del agua.

Para la Figura 2 c) y d), los valores medios de $\mathrm{Cl}^{\text {, }}$ que oscilan entre 200 y $300 \mathrm{mg} / \mathrm{L}$, no presentan una variación importante respecto al valor medio de entrada $(277,3+20,0$ $\mathrm{mg} / \mathrm{L}$, tabla 2). Este resultado es acorde con el obtenido por Aghakhani et al. [13], quienes al tratar de eliminar sales del agua de mar con diferentes materiales adsorbentes (entre los que se encontraban la zeolita y el carbón activado), tampoco obtuvieron eliminación de $\mathrm{Cl}^{-}$, al trabajar con concentraciones afluentes de $1.300 \mathrm{mg} / \mathrm{L}$. Por tanto, y tomando en cuenta lo anterior, se puede concluir que ninguno de los materiales evaluados es capaz de eliminar este compuesto. Para efectos de reutilización, el $\mathrm{Cl}^{-}$ afecta las puntas de las hojas de los cultivos, lo 
que causa un fenómeno denominado necrosis. Al respecto, la NCh 1433 Of. 78 [8] establece como límite máximo un valor de $200 \mathrm{mg} / \mathrm{L}$, y en el mismo sentido, la normativa internacional ha limitado el $\mathrm{Cl}^{-}$a valores por debajo de los 350 $\mathrm{mg} / \mathrm{L}$ [4]. Por tanto, es claro que el uso de este tipo de agua podría generar problemas si se riegan cultivos no tolerantes a este compuesto.

En el caso del sulfato $\left(\mathrm{SO}_{4}^{-2}\right)$ (Figura 2 e) y f)), se puede notar un aumento de entre 20 y $40 \%$ en la concentración efluente a las columnas que utilizaron arena como material filtrante, especialmente durante la primera quincena de operación. Este hecho puede explicarse por la composición mineralógica del material (Tabla 1), en la que se encuentra una proporción de azufre. La presencia de este compuesto en el material, es explicada porque se extrajo de la quebrada Juan Morales, localizada en una zona con gran presencia de minerales al norte de Chile. Por tanto, este resultado muestra que la utilización de materiales filtrantes de la zona, podría llevar a incrementar la concentración de $\mathrm{SO}_{4}^{-2}$ en una etapa inicial. Respecto a los otros materiales filtrantes, no existe un efecto de más de $20 \%$ en aumento o reducción del valor medio de este compuesto. Es importante tener en cuenta que la NCh 1333 Of. 78 [8], establece como límite máximo un valor de 250 $\mathrm{mg} / \mathrm{L}$. En este sentido, tanto el agua afluente como todos los efluentes podrían generar problemas en cultivos regados con esta agua, dada la concentración de este parámetro.

Respecto a los cationes, en el caso del $\mathrm{Ca}^{+2}$, los valores medios evaluados en los efluentes son similares a los del influente con variaciones inferiores a $10 \%$. Esto indica que ninguno de los materiales evaluados, ni la combinación de estos, presenta características de adsorción de este catión. Este resultado es contradictorio al presentado por Aghakhani et al. [13], quienes encontraron que materiales como la zeolita y el carbón activado, en combinación con resinas catiónicas, eran capaces de remover hasta un $60 \%$ de la concentración inicial de $\mathrm{Ca}^{+2}$, que era de alrededor de $16 \mathrm{mg} / \mathrm{L}$. Esta diferencia con el presente estudio podría explicarse, principalmente, porque el uso de las resinas catiónicas podría haber reducido la concentración de $\mathrm{Ca}^{+2}$ hasta valores de concentración que generaron adsorción por parte de la zeolita y el carbón activado. Además, es importante recordar que la concentración media de $\mathrm{Ca}^{+2}$ presente en el afluente (83 $\mathrm{mg} / \mathrm{L}$, Tabla 1), es 5 veces mayor que la del experimento de Aghakhani et al. [13], y, por tanto, sería muy elevada para poder utilizar materiales como la zeolita o el carbón activado para su eliminación.

\subsection{Comportamiento del pH, CE y T}

La Figura 3 presenta la evolución temporal dividida en quincenas, para los valores medios de pH y CE durante el tiempo de operación. En el caso del pH (Figura 3 a) y b)), solo los efluentes a los sistemas de filtración con carbón activado como único medio, o combinado con otro, presentan valores cercanos a 9,0 durante la primera quincena de operación (Figura 2 a) y b)). Al respecto, se ha establecido que, para aguas de riego, los valores de $\mathrm{pH}$ deberían variar entre 6,5 y 8,4 [8-9]. Por tanto, los resultados obtenidos muestran que, a pesar de que el carbón activado es el único medio capaz de retener $\mathrm{B}$, debido al mecanismo de extracción basado en intercambio con iones hidroxilo, este incrementa los valores de $\mathrm{pH}, \mathrm{y}$, por tanto, podría afectar los cultivos irrigados con sus efluentes durante una etapa inicial de operación. Pese a esto, a partir de la segunda quincena se mantiene la eliminación de $B$, sin incrementarse el pH. Esta información sería útil para, en caso tal de aplicar la filtración 

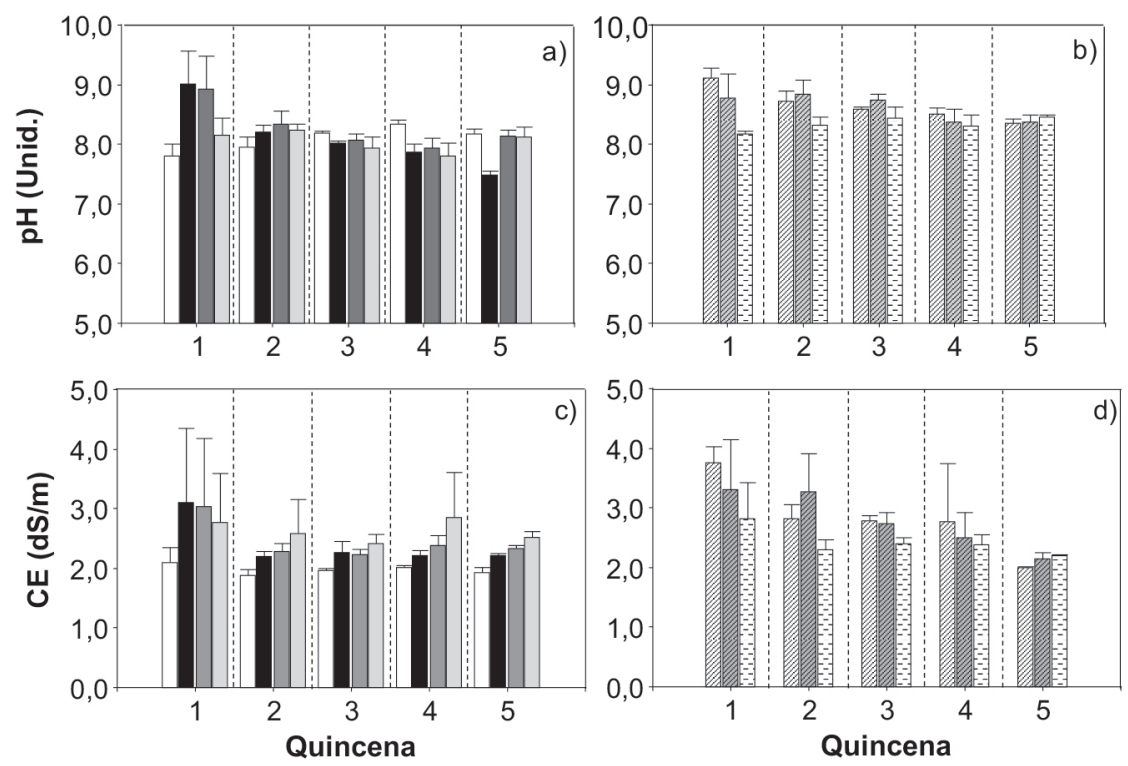

Figura 3. Comportamiento del pH y la CE por quincena. a) y c) filtros de un solo material: $(\square) Z,(\square) C A-A,(\square) C A-B,(\square) A$; b) y d) filtros con dos materiales: (四) Z+CA-B, (四) A+CA-B, (圆) Z+A.

Fuente: Elaboración propia.

con carbón activado, las primeras filtraciones deben ser descartadas como agua de riego.

Respecto a la conductividad eléctrica (CE), dos aspectos son destacables en la Figura 3 c) y d). El primero de estos es el aumento de entre 15 y $60 \%$ de los valores medios medidos en los efluentes a las columnas que utilizaron carbón activado y arena de forma individual o combinada, respecto del afluente, para la primera quincena de operación. En las siguientes quincenas sigue presentándose un incremento, pero este no supera el $20 \%$. En el caso del carbón activado, este aspecto refuerza la recomendación previa de la necesidad de descarte del agua para las primeras filtraciones realizadas.

El segundo aspecto destacable es la reducción de entre 10 y $20 \%$ del valor medio de la CE para los efluentes de la columna que utilizó zeolita como único material filtrante, porcentaje que se mantuvo durante todo el tiempo de operación. Este resultado se explica, ya que ha sido determinado por otros autores que la zeolita tiene capacidad de intercambio catiónico con afinidad de adsorción de cationes, como el $\mathrm{NH}_{4}^{+}$y algunos compuestos metálicos, pero también la zeolita tiene la propiedad de eliminar, mediante intercambio iónico y precipitación, compuestos como el $\mathrm{PO}_{4}^{-3}$ (no evaluados en este estudio) [14-22]. Pese a estas propiedades, este comportamiento no se repite para columnas que combinaron la zeolita con otro material filtrante. Por tanto, no existen efectos sinérgicos por combinar diferentes materiales. Pese a su aporte en la reducción de la CE, los valores nunca estuvieron por debajo de los 1,5 dS/m, y por tanto, este tipo de agua podría ser definida como "agua que puede 
tener efectos adversos en muchos cultivos y necesita de métodos de manejo cuidadosos" [8]. Es importante destacar, también, que estos valores de CE obtenidos son coherentes con la baja o nula eliminación de compuestos, como el cloruro y el sulfato, los que podrían definirse como los principales aportantes para el valor de CE. En este sentido, los sistemas de filtración con materiales porosos, como los propuestos, no serían muy aplicables para reducir la salinidad, y llevaría a la necesidad de utilizar medidas alternativas de bajo costo, como la destilación solar, para la reducción del contenido de sales del agua residual tratada.

Otro de los parámetros físicos evaluados, la temperatura, presenta una similitud entre el afluente y los efluentes de todas las columnas de filtración evaluadas, con valores que oscilan entre 20 y $25^{\circ} \mathrm{C}$, sin que se presente un incremento mayor a $1{ }^{\circ} \mathrm{C}$ en los efluentes. Estos valores oscilan de igual forma que los valores de las temperaturas ambientales de la zona de experimentación, caracterizada como subtropical desértica con influencia marina [23].

\subsection{Comportamiento de otros parámetros de interés en reutilización}

La Tabla 3 resume los resultados obtenidos del monitoreo al día 0 y 60 , de metales pesados y la TAS. En el caso de los metales pesados, estos son importantes en proyectos de reutilización de aguas residuales, primero porque algunos de estos, como el $\mathrm{As}$, el $\mathrm{Pb}$, el $\mathrm{Cd}$ y el $\mathrm{Cu}$, incluso a concentraciones tan bajas como 0,1 mg/L, pueden ser tóxicos para algunas especies de plantas, y segundo, porque algunos de estos, como el Cd, el Cu y el $\mathrm{Zn}$, pueden ser introducidos en la cadena trófica por bioacumulación en los tejidos de las plantas, a niveles tóxicos para sus consumidores [7]. Sin embargo, aguas residuales municipales que tienen tratamiento secundario, normalmente no presentan problemas con estos elementos [7]. En este sentido, la Tabla 3 muestra cómo los metales pesados evaluados presentan valores por debajo de lo exigido en la NCh 1333 Of. 78, con la única excepción del As (2 casos) y $\mathrm{Mn}$ (1 caso) para los efluentes de columnas que incluían arena [8]. Al respecto, ha sido ampliamente documentada la contaminación natural por As que existe en el norte de Chile, y que, como puede verse en este experimento, se puede lixiviar cuando materiales de la zona son utilizados con fines de filtración [24].

Por su parte, la TAS presenta un incremento por sobre 9 (adimensional), en las columnas que utilizan carbón activado y arena, como medio filtrante, únicamente para el día 0 de experimentación. Esto de nuevo corresponde con lo argumentado previamente, sobre el efecto inicial que origina el carbón activado en el efluente, y los problemas sobre su uso en riego de actividades agrícolas. En el caso de la arena, nuevamente evidencia los problemas derivados de lixiviación que pueden originarse, y que para este parámetro se podrían asociar a los contenidos de sodio $(2,71 \%$, Tabla 1$)$ que presenta este material. Otro aspecto que es destacable, es la reducción a valores inferiores a 5 para la TAS en los efluentes de las columnas que utilizan zeolita como medio filtrante único o combinado con arena.

La TAS resulta un parámetro importante en la reutilización deaguas residuales municipales en actividades de riego, debido a que valores sobre 9 traen consigo problemas relacionados con la estructura del suelo, que afecta la aireación e infiltración de los mismos, especialmente cuando se aplica riego por inundación [9]. Sin embargo, solo el análisis conjunto de la TAS y la CE definirá el potencial grado de daño que puede ser causado. Tomando en cuenta esto, 
Tabla 3. Concentraciones de algunos metales incluidos en la NCh 1333 Of. 78 evaluados al día 0 (0 d) y día 60 (60 d) de operación de los sistemas de filtración

\begin{tabular}{|c|c|c|c|c|c|c|c|c|c|c|c|c|c|c|c|}
\hline \multirow[b]{2}{*}{ Parámetro } & \multicolumn{2}{|c|}{ z } & \multicolumn{2}{|c|}{ CA-A } & \multicolumn{2}{|c|}{ CA-B } & \multicolumn{2}{|c|}{ A } & \multicolumn{2}{|c|}{$Z+C A-B$} & \multicolumn{2}{|c|}{$A+C A-B$} & \multicolumn{2}{|c|}{$Z+A$} & \multirow[b]{2}{*}{$\begin{array}{l}\text { NCh } 1333 \\
\text { Of. } 78 \text { [8] }\end{array}$} \\
\hline & od & $60 \mathrm{~d}$ & od & $60 \mathrm{~d}$ & od & $60 \mathrm{~d}$ & od & $60 \mathrm{~d}$ & od & $60 \mathrm{~d}$ & od & $60 \mathrm{~d}$ & od & $60 \mathrm{~d}$ & \\
\hline As (mg/L) & $<0,001$ & 0,006 & 0,02 & 0,019 & 0,03 & 0,021 & $\underline{0,17}$ & 0,01 & 0,05 & 0,004 & $\underline{0,1}$ & 0,062 & 0,04 & 0,038 & 0,1 \\
\hline $\mathrm{Cd}(\mathrm{mg} / \mathrm{L})$ & $<0,01$ & $<0,01$ & $<0,01$ & $<0,01$ & $<0,01$ & $<0,01$ & $<0,01$ & $<0,01$ & $<0,01$ & $<0,01$ & $<0,01$ & $<0,01$ & $<0,01$ & $<0,01$ & 0,01 \\
\hline $\mathrm{Cu}(\mathrm{mg} / \mathrm{L})$ & $<0,01$ & $<0,01$ & 0,01 & $<0,01$ & 0,02 & 0,02 & 0,13 & $<0,01$ & 0,02 & $<0,01$ & 0,03 & 0,04 & 0,04 & 0,06 & 0,2 \\
\hline $\mathrm{Fe}(\mathrm{mg} / \mathrm{L})$ & 0,02 & 0,04 & 0,03 & 0,06 & 0,02 & 0,67 & 0,01 & 0,05 & $<0,01$ & $<0,01$ & 0,01 & 0,06 & $<0,01$ & 0,03 & 5,0 \\
\hline $\mathrm{Mn}$ (mg/L) & 0,12 & 0,03 & $<0,01$ & $<0,01$ & $<0,01$ & $<0,01$ & 0,02 & 0,1 & $<0,01$ & $<0,01$ & $<0,01$ & 1,08 & $<0,01$ & 0,59 & 0,2 \\
\hline $\mathrm{Pb}(\mathrm{mg} / \mathrm{L}$ & $<0,05$ & $<0,05$ & $<0,05$ & $<0,05$ & $<0,05$ & $<0,05$ & $<0,05$ & $<0,05$ & $<0,05$ & $<0,05$ & $<0,05$ & $<0,05$ & $<0,05$ & $<0,05$ & 5,0 \\
\hline $\mathrm{Zn}$ (mg/L) & 0,07 & 0,04 & 0,03 & 0,07 & 0,06 & 0,07 & 0,04 & 0,06 & 0,04 & 0,03 & 0,03 & 0,04 & $<0,01$ & 0,04 & 2,0 \\
\hline TAS & 1 & 0,7 & 14 & 9,2 & 13 & 9 & 10 & 6,4 & 8,5 & 2,8 & 14 & 7,7 & 1,9 & 3,1 & $>9$ \\
\hline
\end{tabular}

Fuente: Elaboración propia. TAS: Tasa de Adsorción de Sodio. La TAS es un parámetro adimensional.

y lo presentado por Ayers y Westcot [6], se podría indicar de forma general que el agua afluente y el efluente de todos los materiales filtrantes utilizados, a pesar de los altos valores de TAS y CE para algunos de estos, en general no presentarían problemas con los suelos. Pese a esto, en el caso del norte de Chile la presencia de elevadas concentraciones de As (53 a $448 \mathrm{mg} / \mathrm{kg}$ ) y otros elementos como el B [25], podrían generar otros tipos de problemas en conjunción con el agua evaluada.

En este contexto, alternativas de uso de sistemas de cultivo sin suelo, como la hidroponía y la aeroponía [26], y sistemas de riego para estas, serían interesantes de evaluar a futuro. Esto porque, algo que ha demostrado este estudio, es que si bien el agua residual municipal tratada debido al alto grado de cobertura de tratamiento presente en Chile (> $90 \%$, [5]) representa una fuente interesante de agua dulce, está claro que podrían presentarse problemas con el alto contenido de sales $(>1,5$ $\mathrm{dS} / \mathrm{m})$, y que de todos los materiales evaluados solo la zeolita natural tiene el potencial de ser aplicado como medio filtrante. 


\section{CONCLUSIONES}

El agua residual municipal tratada, utilizada en este estudio, fue clasificada como típica de zonas áridas, lo que representa un elevado contenido de sales (CE $>1,5 \mathrm{dS} / \mathrm{m}$ ). Los resultados mostraron que el carbón activado es capaz de remover boro a valores por debajo de $0,75 \mathrm{mg} / \mathrm{L}$. Sin embargo, en una etapa inicial (15 días) el efluente filtrado presentó valores de CE y pH superiores a 2,5 dS/m y 8,4 unidades, respectivamente, lo que es probable que podría afectar su aplicación en riego de cultivos y de plantas en general. El uso de la arena, procedente de la región, por el alto contenido de arsénico y sodio, limitó su potencial uso como material filtrante. Si se utiliza una arena diferente, dependiendo de su origen, no debería generar aporte a ninguno de los parámetros de calidad de agua evaluados. La zeolita, sin mezcla con otro material filtrante, mostró ser la mejor opción para reducir la salinidad del agua, lo que se reflejó en una disminución de la CE de hasta 20 $\%$ sin afectar los otros parámetros de calidad de agua evaluados en este estudio, y además, no mostró un estado de saturación durante el tiempo de operación evaluado. Finalmente, para las columnas que utilizaron mezcla de materiales filtrantes, no pudo establecerse un efecto sinérgico para la eliminación de los parámetros de calidad del agua evaluados en este estudio.

\section{AGRADECIMIENTOS}

Este trabajo fue financiado por CONICYT REGIONAL/ GORE TARAPACÁ/ CIDERH a través del fondo No. R09l1001. Además, agradecer a la empresa sanitaria regional (Aguas del Altiplano), y especialmente a Sergio Fuentes, Renzo Aravena e Isabel Tello, por el acceso al afluente usado y la analítica aportada para el experimento. También, agradecimientos al laboratorio de Bioarqueología del Instituto de Alta Investigación de la Universidad de Tarapacá en cabeza del Dr. Bernardo Arriaza, por facilitar el trabajo de caracterización de los materiales. Finalmente, a Fernando Arancibia, Wladimir Chávez y Camila Jorquera por la colaboración en el montaje de las unidades experimentales.

\section{BIBLIOGRAFÍA}

[1] Norton-Brandão, D., Scherrenberg, S., Van Lier, J. (2013). Reclamation of used urban waters for irrigation purposes - A review of treatment technologies. Journal of Environmental Management, 122, pp. 85-98.

[2] Ministerio de Obras Públicas (MOP). (2013). Estrategia Nacional de Recursos Hidricos. En: http://www.mop.cl/ Documents/ENRH_2013_OK.pdf (01 de noviembre de 2013).

[3] United States Environmental Protection Agency (Usepa). (2012). Guidelines for Water Reuse. EPA/600/R-12/618. Washington D.C, USA: Usepa.

[4] Pedrero, F., Kalavrouziotis, I., Alarcón, J., Koukoulakis, P., Asano, T. (2010). Use of treated municipal wastewater in irrigated agriculture-Review of some practices in Spain and Greece. Agricultural Water Management, 97, pp. 1233-1241.

[5] Superintendencia de Servicios Sanitarios (SISS). (2013). Plantas de tratamiento de aguas servidas en operación. En: http://www.siss.gob.cl/577/w3- 
propertyvalue-3544.html (01 de noviembre de 2013).

[6] Ayers, R., Westcot, D. (1985). Water Quality for Agriculture. Food and Agriculture Organization of the United Nations (FAO). En: http://www.fao.org/DOCReP/003/ T0234e/T0234E00.htm\#TOC (01 de junio de 2015).

[7] Asano, T., Burton, F., Leverenz, H., Tsuchihashi, R., Tchobanoglous, G. (2007). Water Reuse: Issues, Technologies, and Applications. New York, USA: McGrawHill, pp. 1570.

[8] Instituto Nacional de Normalización (INN). (1978). Norma Chilena (NCh) 1333 Of. 78 modificada 1987. Requisitos de calidad del agua para diferentes usos. Santiago de Chile: INN.

[9] Lazarova, V., Bahri, A. (2004). Water Reuse for Irrigation: Agriculture, Landscapes, and Turf Grass. Boca Raton, USA: CRC Press, pp. 432.

[10] Cáceres, L., Delatorre, J., De la Riva, F., Monardes, V. (2003). Greening of Arid Cities by Residual Water Reuse: $A$ Multidisciplinary Project in Northern Chile. Ambio, 32, pp. 264-268.

[11] Castro, E., Mañas, M., De Las Heras, J. (2011). Effects of wastewater irrigation on soil properties and turfgrass growth. Water Science and Technology, 63, pp. 1678-1688.

[12] Aghakhani, A, Mousavi S., MostafazadehFard B. (2013). Desalination of saline water with single and combined adsorbents. Desalination and Water Treatment, 51, pp. 1928-1935.
[13] Aghakhani A., Mousavi S., MostafazadehFard B., Rostamian R., Seraji M. (2011). Application of some combined adsorbents to remove salinity parameters from drainage water. Desalination, 275, pp. 217-223.

[14] Wang, S., Peng, Y. (2010). Natural zeolites as effective adsorbents in water and wastewater treatment. Chemical Engineering Journal, 156, pp. 11-24.

[15] Lakoleva, E., Sillanpaa, M. (2013). The use of low-cost adsorbents for wastewater purification in mining industries. Environmental Science and Pollution Research, 20, pp. 7878-7899.

[16] Brix, H., Arias, C., del Bubba, M. (2001). Media selection for sustainable phosphorus removal in subsurface flow constructed wetlands. Water Science and Technology, 44, pp. 47-54.

[17] Arias, C., Brix, H. (2005). Phosphorus removal in constructed wetlands: can suitable alternative media be identified? Water Science and Technology, 51, pp. 267-273.

[18] United States Environmental Protection Agency (Usepa). (1999). Folleto informativo de tecnología de aguas residuales filtros intermitentes de arena. En: http://water.epa.gov/aboutow/owm/ upload/2004_07_07_septics_septic_ fs_intermittent_sp.pdf (01 de diciembre de 2013).

[19] American Public Health Association (APHA), American Water Works Association (AWWA), Water Environment Federation (WEF) (APHA-AWWA- 
WEF). (1998). Standard methods for the examination of water and wastewater (20th edition). Washington D.C., USA: APHA-AWWA-WEF.

[20] Instituto Nacional de Normalización (INN). (1996). Parte 9: Determinación de arsénico - Método de espectrofotometría de absorción atómica con generación continua de hidruros. Parte 10: Determinación de metales pesados - Método de espectrofotometría de absorción atómica con llama. Aguas residuales - Métodos de análisis. Santiago de Chile: INN.

[21] Türker, O., Vymazal, J., Türe, C. (2014). Constructed wetlands for boron removal: A review. Ecological Engineering, 64, pp. 350-359.

[22] Vera, I., Araya, F., Andrés, E., Sáez, K., Vidal, G. (2014). Enhanced phosphorus removal from sewage in mesocosmscale constructed wetland using zeolite as medium and artificial aeration. Environmental Technology, 35, pp. 16391649.
[23] Centro de Investigación y Desarrollo en Recursos Hídricos (Ciderh). (2013). Recursos hídricos - Región de Tarapacá - Diagnóstico y sistematización de la información. lquique, Chile: Editorial Universidad Arturo Prat.

[24] Bundschuh, J., Litter, M., Parvez, F., RománRoss, G., Nicolli, H., Jean, J., Liu, C., López, D., Armienta, M., Guilherme, L., Cuevas, A., Cornejo, L., Cumbal, L., Toujaguez, R. (2012). One century of arsenic exposure in Latin America: A review of history and occurrence from 14 countries. Science of the Total Environment, 429, pp. 2-35.

[25] Tapia, Y., Díaz, O., Pizarro, C., Segura, R., Vines, M., Zúñiga, G., Moreno-Jiménez, E. (2013). Atriplex atacamensis and Atriplex halimus resist as contamination in PreAndean soils (northern Chile). Science of the Total Environment, 450-451, pp. 188196.

[26] Urrestarazau, M. (2004). Tratado de cultivo sin suelo. Madrid, España: Ediciones Mundi Prensa, pp. 914. 